\title{
MAGNETIC STRUCTURES IN ULTRATHIN FILMS AND NANOSTRUCTURES: SIMULATION
}

\author{
J.-C.S. LÉVY ${ }^{a *}$, A. GHAzALI ${ }^{b}$ AND E.YU. VedMEDEnKo ${ }^{c}$ \\ ${ }^{a}$ LPTMC, case 7020, Université Paris 7, 75251 Paris 5, France \\ ${ }^{b}$ GPS, UMR-7588 CNRS, Universités Paris 7 et Paris 6, 75251 Paris 5, France \\ ${ }^{c}$ MPI für Mikrostrukturphysik, 06120 Halle, Germany
}

In magnetic ultrathin films and dots, competing exchange, anisotropy and dipolar couplings lead to a large variety of magnetic structures. These structures are obtained by means of the Monte Carlo simulations. Three classes of magnetic structures are obtained according to anisotropy-to-dipolar energy ratio: Ising striped structures, $X Y$-spin structures with vortices, and twisted spin phases at the spin reorientation transition. Domain wall nucleation and motion at the coercive field are also accessible.

PACS numbers: 75.70.Ak, 75.50.Tt, 75.60.Ch, 75.40.Mg

\section{Introduction}

Ultrathin films of a single atomic monolayer or of a few monolayers deposited over non-magnetic materials are now available for magnetic experiments [1]. Similarly nanostructures such as ultrafine magnetic particles have been obtained by different methods such as electrochemistry, or soft chemistry route such as the polyol process [2], or by nanolithography [3], or by aggregated atomic beams. These new materials are interesting both by their original properties and by the high density of independent objects they generate.

\section{Magnetic interactions, model, and simulation}

The magnetic structures of these nanomaterials result from the competition between exchange interaction $J$ with nearest spins, magnetocrystalline anisotropy, long-range dipolar coupling $D$ between spins and Zeeman interaction with the external magnetic field $\boldsymbol{H}$. Here we assume a uniaxial first-order anisotropy $K$. The Hamiltonian with vector spins $\boldsymbol{S}_{\boldsymbol{i}}$ reads

*e-mail: levy@ccr.jussieu.fr 


$$
\begin{aligned}
H= & -J \sum_{\langle i j\rangle} S_{i} \cdot S_{j}-K \sum_{i} S_{i, z}^{2}+D \sum_{i j}\left[\frac{S_{i} \cdot S_{j}}{r_{i j}^{3}}-3 \frac{\left(S_{i} \cdot r_{i j}\right)\left(S_{j} \cdot r_{i j}\right)}{r_{i j}^{5}}\right] \\
& -\boldsymbol{H} \cdot \sum_{i} S_{i} .
\end{aligned}
$$

These magnetic structures are expected to be complex due to the long-range dipolar interactions which yield antagonistic forces on the spins at all length scales. In addition, the resulting magnetic patterns depend on the shape of the sample. Therefore, to account for such structures, all dipolar couplings between spins in the sample should be included.

The aim of this paper is to classify these magnetic structures in a wide range of parameter values by analyzing the various magnetic orders obtained from the Monte Carlo (MC) simulations after annealing and slow cooling. Simple scaling arguments based on the values of the dimensionless ratio $R=D / J a^{3}$ with the lattice parameter $a$, together with a consistent rescaling of the other Hamiltonian coupling parameters, allow to consider spin blocks of various sizes instead of single spins [4]. This is a very useful procedure since it permits to account for large sample patterns with a relatively limited number of effective spins.

Extensive Monte Carlo simulations were performed. Starting at a high enough temperature, different temperatures are explored in a slow cooling process in order to deduce the low temperature configurations. This is a very long process because of the intrinsic all range frustration due to dipolar couplings.

A useful criterion for selecting low temperature configurations is the energy comparison between relaxed $\mathrm{MC}$ configurations and hypothetic configurations suggested from symmetry or from simple considerations [4]. Such a control strongly helps in rationalizing the choice of optimal configurations, given the high degree of approximate degeneracy which makes the exact ground state very difficult to single out. Since the spin patterns are size- and shape-dependent, free boundary conditions are used. This also precludes any periodicity artificially induced by periodic boundary conditions [4].

Demagnetizing fields are due to dipolar interactions and are well known to be shape-dependent. Thus different sample shapes were considered in order to settle this classification on a clear basis. For ultrathin films, square, rectangle, or disk-shaped samples were considered with two lattice types: the triangular lattice represents a (111)-oriented fcc lattice surface or a surface normal to the hcp-lattice $c$-axis and the square lattice represents a (001)-oriented fcc-lattice surface. For dots, hcp-lattice spheres of different sizes were used. In our MC simulations, most samples contain about 10000 vector spins. The maximum size studied is about 50000 vector spins.

For all samples, three general classes of magnetic structures must be distinguished: (i) with a very strong anisotropy, perpendicular to the film or along the dot $c$-axis, an Ising-like spin structure is formed in which the magnetization is parallel to this direction; (ii) with a weak anisotropy energy as compared to dipolar energy, a planar $(X Y)$ vector spin structure appears at low temperature in the film plane or in the plane normal to the $c$-axis of the dot; (iii) in the intermediate case where the perpendicular anisotropy energy balances the dipolar energy, spins 
are evenly oriented in all directions. At this point, a spin reorientation transition occurs. In the following, we describe these different spin patterns in some detail.

\section{Ultrathin films}

For very thin films without external field, the low temperature structures are: (i) With a strong perpendicular anisotropy (Ising-type spins), magnetic domains with up-spin and down-spin alternate stripes of equal width are arranged in Chevry patterns. On a large scale, these domains define mazes [5]. Similar structures are already well known to occur for thicker samples [6]. (ii) With a weak anisotropy, the low temperature configurations consist in planar ( $X Y$-type) spins with clockwise and anticlockwise vortices [4]. These vortices are rather uniformly distributed over the sample as seen in Fig. 1. This new feature is not yet fully observed in ultrathin films, presumably because of the intrinsic difficulty to observe in-plane vector spin patterns at the nanometre scale. However, similar features were experimentally observed in magnetic-non-magnetic multilayers by the Lorentz microscopy [7]. (iii) With an intermediate uniaxial anisotropy, i.e., around the reorientation transition, a modulated twisted spin configuration takes place and includes almost in-plane spin domains surrounding patches of out-of-plane spins. The latter ones are arranged as twisted spin bunches as seen in Fig. 2. Experimentally, the spin reorientation transition is now well known to occur as a function of various parameters, such as layer thickness, temperature, etc. [1], but the spin reorientation just starts to be observed at the nanometre scale [8] with results similar to those predicted here [5].

The magnetic structures described above are very sensitive to the action of an external field. (i) In the Ising case, stripes with spins parallel to the field

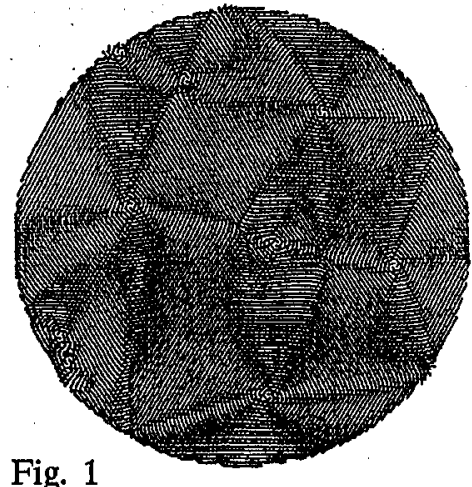

Fig. 1

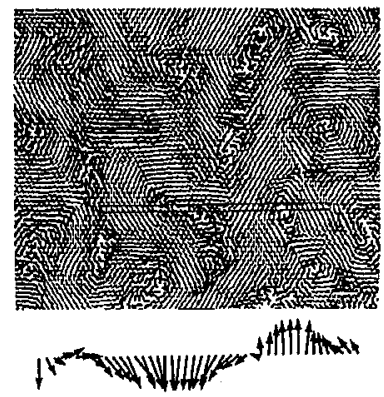

Fig. 2

Fig. 1. Low-temperature in-plane spin pattern with vortices for a disk-shaped sample of 10192 spins on a triangular lattice. $D / J a^{3}=1$.

Fig. 2. Spin reorientation effects. Portion of a disk of 10192 spins on a triangular lattice. Thin arrows are nearly in-plane spins. Thick arrows are out-of-plane spins, either above (black) or below (grey). Bottom: spin profile in the vertical plane along the indicated segment. $D / J a^{3}=0.1$ and $K / J=0.9$. 
start to widen out, with local indentations, at the expense of antiparallel spin stripes. As the external field is increased further, magnetic bubble domains appear until the magnetic saturation is reached. The bubbles remain in a metastable state after the field has been removed, just as in the case of thicker samples [6]. (ii) With vector spins, when an in-plane external field is increased up to the coercive value, $360^{\circ}$ walls nucleate at the sample edge and move through the sample as solitons which can be followed step by step during the MC calculations. This nucleation-propagation process is a quite general problem [9]. The study of the dynamics of this complex motion is in progress.

\section{Magnetic dots}

Let us now turn to the magnetic dots. Without external field, the low temperature structures are: (i) For a large uniaxial anisotropy (the Ising spins), the partition between up-spin and down-spin domains has a rather complex geometry. (ii) For a small anisotropy, $X Y$-spin structures develop in the densest planes with vortices. The core-vortex line is a skew line crossing the sample. (iii) With a moderate anisotropy, complex three-dimensional magnetic structures appear that contain zones with spins parallel to the densest planes and other zones with spins normal to these planes. In between, the spins rotate on short distances.

The magnetic structure of dots in presence of a field is being processed now with preliminary results which are comparable to those obtained for ultrathin films, i.e., three-dimensional wall nucleation and wall motion with distortion.

As a conclusion we emphasize the interest of the new magnetic structures described here. For instance, in ultrathin layers, magnetic vortices and twisted spin configurations are expected to occur, and preliminary observations of similar structures exist [8]. On the other hand, both stripe structures and vortex structures were observed in magnetic dot arrays [3]. Another point of interest is the fast soliton dynamics which is shown to occur at the coercive field. This is crucial for recording applications and critical properties of this motion are now extensively studied experimentally and theoretically.

\section{References}

[1] R. Allenspach, J. Magn. Magn. Mater. 129, 160 (1994).

[2] P. Toneguzzo, G. Viau, O. Acher, F. Fiévet-Vincent, F. Fiévet, Adv. Mater. 13, 1032 (1998).

[3] M. Hehn, K. Ounadjela, J.-P. Bucher, F. Rousseaux, D. Decanini, B. Bartenlian, C. Chappert, Science 272, 1782 (1996).

[4] E.Yu. Vedmedenko, A. Ghazali, J.-C.S. Lévy, Phys. Rev. B 59, 3329 (1999).

[5] E.Yu. Vedmedenko, A. Ghazali, J.-C.S. Lévy, Surf. Sci. 402-404, 391 (1998).

[6] J.A. Cape, G.W. Lehman, J. Appl. Phys. 42, 5732 (1971).

[7] A.C. Daykin, J.P. Jakubovics, J. Appl. Phys. 80, 3408 (1996).

[8] T. Duden, E. Bauer, Phys. Rev. Lett. 77, 2308 (1996).

[9] M.E. Gouvêa, G.M. Wysin, S.A. Leonel, A.S.T. Pires, T. Kamppeter, F.G. Mertens, Phys. Rev. B 59, 6229 (1999). 